\title{
Fucoidan induces Apoptosis in MDA-MB-231 Cells by Activating Caspase Cascade and Down-regulating XIAP
}

\author{
Amal M. Banafa, Sadia Roshan, Yunyi Liu (柳昀熠), Shasha Zhao(赵沙沙), \\ Guangxiao Yang (杨广笑), Guangyuan He (何光源), Mingjie Chen (陈明洁: \\ 通讯作者)井
}

The Genetic Engineering International Cooperation Base of Ministry of Science and Technology, Key Laboratory of Molecular Biophysics of Chinese Ministry of Education, College of Life Science and Technology, Huazhong University of Science \& Technology (HUST), Luoyu Road 1037, Wuhan, Hubei, 430074, P.R. China

\begin{abstract}
Summary: Previous studies have shown fucoidan could induce apoptosis in breast cancers, while the detailed mechanisms need to be investigated.Results of this study illustrated that number of viable MDA-MB-231 cells was decreased by fucoidan with a dose-dependent manner, while the fucoidan did not affect the viable epithelial cells number of normal human mammary (MCF10A).. After incubation with fucoidan, several apoptotic events such as chromatin condensation and increase of the population of G2/M phase were observed in MDA-MB-231 cells. RT-PCR and Western blot results showed that the activation of caspase cascade such caspase-3, caspase-8 and caspase-9, as well as the expression of XIAP (X-linked inhibitor of apoptosis protein), was involved in the apoptosis process. Besides that, reactive oxygen species(ROS) production was increased after fucoidan treatment. These results indicated that fucoidan could induce the apoptotic cell death through modulation of $X I A P$ and caspases activation in MDA-MB-231 cells and could also induce the generation of ROS.
\end{abstract}

Keywords: Fucoidan, Apoptosis, MDA-MB-231 Cells, Caspase Cascade, XIAP, reactive oxygen species (ROS).

\section{Introduction}

Natural dietary compounds have been widely and safely consumed over centuries, and preclinical studies suggest that most of them have potential applications in pharmacology and cancer therapy. Fucoidan, a class of fucose-enriched sulfated polysaccharides, is a naturally occurring polysaccharide compound in brown alga, such as Fucus vesiculosus, Cladosiphon okamuranus and Laminaria saccharina ${ }^{[1,2]}$. It has recently been reported that fucoidan possesses a wide variety of biological activities, including anticoagulant, antiviral, antiangiogenic, anticancer and immune-modulatory activities ${ }^{[3]}$. Studies in vitro have indicated that fucoidan exhibits protection effect against various cancers, including human lymphoma, promyelocytic leukemia, colon carcinoma, breast carcinoma, hepatoma and melanoma. Several investigations have demonstrated that fucoidan can effectively suppress proliferation and colony formation of cancer cells in vitro ${ }^{[4]}$.

Apoptosis, or programmed cell death, is an important aspect of chemotherapy-induced tumor cell death and also the major mechanism of tumor cell death induced by many anticancer drugs and natural products ${ }^{[5]}$. Caspase-dependent apoptosis is characterized by activation of either the extrinsic pathway, initiated by activation of death receptors leading to the cleavage of caspase- 3 and -8 , or the intrinsic pathway, triggered by mitochondrial depolarization, release of cytochrome $c$ and the subsequent activation of caspase-9 ${ }^{[6,7]}$. Disruption to the factors regulating these apoptotic pathways contributes substantially to the transformation of a normal cell into a tumor cell, and the cells of some tumor types are relatively resistant to apoptosis ${ }^{[8,9]}$.

Significant effect of fucoidan has been shown to be associated with both decreased proliferation and increased apoptosis. Mechanisms associated with apoptosis have been described, including production of oxidative stress and activation/expression of modulation proteins, such as caspases-3, -8, -9, XIAP, CDK1 and cyclin B.

Caspases are aspartate-specific cysteine proteases that are instrumental in the apoptotic process. They exist in all nucleated cells as inactive zymogens and are activated by proteolytic cleavage. Caspases are categorized into initiators and effectors on the basis of their positions in apoptotic proteolytic cascades. Initiator caspases trigger and amplify apoptosis by activating effector caspases and pro-apoptotic factors. Effector caspases act at cytoplasmic sites, and eventually at other organelles, including the nucleus, to execute the proteolytic program that finalizes cell destruction.

Caspase- 3 is a caspase protein that interacts with caspase- 8 and -9. It is encoded by the CASP3 gene. The caspase- 3 removes the IBM (The tethering site) of caspase- 9 by proteolysis at Asp ${ }^{330}$, thereby denying XIAP the ability to efficiently inhibit caspase-9. XIAP (X-linked inhibitor of apoptosis protein), is one from many members of the IAP (inhibitor of apoptosis protein) family capable of binding amino acid sequences 
similar to that of the neo-N-terminus of caspase-9, most of which are characterized as having an alanine residue in the first position and often a proline residue in the third position.

ROS are considered to be an apoptotic death signal ${ }^{[6]}$. However, low physiological levels of ROS also serve as a signaling messenger to mediate various biological responses, including cell proliferation, angiogenesis, innate immunity, gene expression, apoptosis and senescence ${ }^{[7]}$. It has also been established that increased levels of these short-lived reactive molecules can exert harmful effects by inducing oxidative damage to biological macromolecules and disrupting the cellular reduction-oxidation (redox) balance. Such disturbances to ROS homeostasis are generally considered to be a risk factor for the initiation and progression of diseases such as atherosclerosis, neurodegeneration and cancer ${ }^{[8]}$. ROS induce depolarization of the mitochondrial membrane potential (MMP, $\Delta \psi \mathrm{m}$ ) and the release of cytochrome c from the mitochondria into the cytosol, where cytochrome $\mathrm{c}$ triggers activation of caspase- 9 and initiates the caspase cascade, which ultimately induces the cell to undergo apoptosis ${ }^{[9]}$. Tumor cells are more sensitive to fluctuations in the levels of ROS than normal cells; therefore, ROS are also considered as an important target in anticancer drug research ${ }^{\text {[10], [11]. }}$.

There are two major pathways involved in the process of the apoptosis mediation. These pathways are the death receptor pathway (extrinsic) and the mitochondrial pathway (intrinsic). Stimulation of death receptors in the extrinsic pathway such as fatty acid synthase (FAS ), leads to clustering and formation of death-inducing signaling complex, which includes the adaptor protein Fas-associated death domain (FADD) and initiator caspases, such as caspase- 3 and -8 . The activated caspases- 8 directly activates downstream effector caspases, such as caspase- $3^{[12]}$. This study illustrated that apoptosis in breast cancer MDAMB-231 cells is induced by fucoidan without affecting the viability of normal epithelial cells mediated by extrinsic pathway. In fact, caspase- 3 and -8 are regarded as essential for fucoidan-induced apoptosis. Therefore we examined whether FAS could protect against apoptosis induced by agents that stimulate the extrinsic pathway ${ }^{[12,13]}$.

Breast cancer is one of the most common human malignancies and the second leading cause of cancerrelated deaths in women, and its incidence in the developing world is on the rise ${ }^{[14]}$. Different treatment strategies have been employed to reduce the mortality rate, including surgery, radiotherapy, and adjuvant chemo

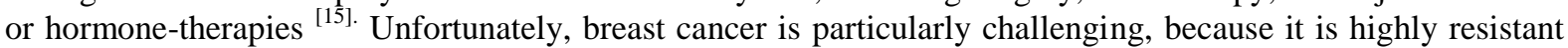
to radiation and conventional chemotherapeutic agents, and such resistance is associated with a poor prognosis for this metastatic disease, particularly in hormone receptor-positive breast cancer ${ }^{[14]}{ }^{[16]}$. About $30-40 \%$ of women with this form of cancer will develop metastasis and eventually die from this disease ${ }^{[17]}$. Novel therapeutic agents are therefore needed to deal with the increasing incidence of human breast cancer. The MDAMB-231 cell line is an estrogen receptor alpha $(\mathrm{ER} \alpha)$ negative human breast cancer cell line, which was obtained from a patient in 1973 at M. D. Anderson Cancer Center. With epithelial-like morphology, the MDAMB-231 cells appear phenotypically as spindle shaped cells as shown in Fig 1.

\subsection{Chemicals}

\section{Materials and Methods}

Fucoidan was purchased from Sigma (USA). Dulbecco's Modified Eagle's Medium (DMEM) medium was obtained from Gibco (USA). Annexin V-FITC/PI assay kit was purchased from Beijing Zoman Biotechnology Co., Ltd (China). Hoechst 33258 was purchased from Beyotime Institute of Biotechnology (China). Fetal bovine serums (FBS) and phosphate-buffered saline (PBS) were purchased from Invitrogen (USA). Dimethylsulfoxide (DMSO), Propidiumiodide (PI), TritonX-100, 3- (4, 5-dimethyl-thiazol-2-yl) -2, 5diphenyltetrazoliumbromide (MTT) and ethylene diaminetetraacetic acid (EDTA) were purchased from Sigma (USA). Monoclonal antibodies against GAPDH, cyclin B1, CDK1, Bax, and Bcl-2 were purchased from Santa Cruz Biotechnology (USA). The secondary horseradish peroxidase (HRP) linked antibodies were obtained from Wuhan Boster Biological Engineering Co. Ltd., China. Alcohol is from Wuhan Anke Biological Technology Co. Ltd.

\subsection{Cell Culture}

MDA-MB-231 cell line and the control cell line MCF-A10, obtained from the China Center Type Culture Collection (China), were cultured in DMEM medium supplemented with FBS, 100 units/ml of penicillin and $100 \mathrm{mg} / \mathrm{ml}$ of streptomycin. Cells were maintained at $37{ }^{\circ} \mathrm{C}$ in a humidified atmosphere of $5 \% \mathrm{CO}_{2}$ in air.

\subsection{MTT Assay}

The effects on cell proliferation were measured by MTT assay, based on the ability of live cells to convert tetrazolium salt into purple formazan. In brief, the cells were seeded in 96-well microplates and incubated overnight. Then the cells were treated with different concentrations of the fucoidan $(0,0.2,0.4,0.6$, 0.8 and $1 \mathrm{mg} / \mathrm{ml}$ ) for $24 \mathrm{~h}$. At the end of these periods, $20 \mu \mathrm{l}$ of MTT stock solution $(5 \mathrm{mg} / \mathrm{ml}$, Sigma, St. Louis, MO) were added to each well and the plates were further incubated for $4 \mathrm{~h}$ at $37^{\circ} \mathrm{C}$. The supernatant was removed and $100 \mu \mathrm{l}$ of DMSO were added to each well to solubilize the water-insoluble purple formazan 
crystals. The absorbency at a wavelength of $570 \mathrm{~nm}$ was measured with Multiscan MCC 340 microplate reader (Titertek, Huntsville, AL). All the measurements were performed in triplicate. Results are expressed as the \% proliferation with respect to fucoidan-treated cells.

\subsection{Cell cycle analysis}

Cells were treated with fucoidan for $24 \mathrm{~h}$ at different concentrations. Then, the cells were collected by trypsinization and washed once with PBS. The cells were fixed with $80 \%$ ethanol for $2 \mathrm{~h}$ at $-20{ }^{\circ} \mathrm{C}$. Then the cells were collected by centrifugation at $15,000 \mathrm{rpm}$ for $5 \mathrm{~min}$ and then resuspended in PBS containing $10 \mu \mathrm{g} / \mathrm{mL}$ PI. After incubation at $37^{\circ} \mathrm{C}$ for $30 \mathrm{~min}$ in dark,the DNA contents of the cells were analyzed using Cytomics TM FC 500 Flow Cytometer (Beckman, USA). The data were analyzed using the Modi FIT program.

\subsection{AnnexinV-FITC/PI Assay}

The annexinV-PI double-staining method was used. MDA-MB-231 cells were seeded into 6 well plates $\left(5 \times 10^{3}\right.$ cells/well) and cultured for $24 \mathrm{~h}$. After treatment with or without fucoidan for varying concentrations, the cells were stained with the annexin V-FITC labeling solution (annexin V-fluorescein in a binding buffer containing PI). The plates were further incubated for $15 \mathrm{~min}$ in the dark, and then images of the cells were acquired using Cytomics TM FC 500 Flow Cytometer (Beckman, USA). The cells with apoptotic morphology of nuclei condensation, or annexin V-positive cells were analyzed using the flow Jo 7.6.1 software.

\subsection{Hoechest Test}

The induction of apoptosis by fucoidan in MDA-MB-231 cells was confirmed in fluorescence photomicrographs of cells stained with Hoechst 33258 at dose depending manner.

\subsection{Reverse Transcription-Polymerase Chain Reaction (RT-PCR)}

Total RNA was extracted from MDA-MB-231 cells using the RNA Extraction Kit (Beijing Zoman Biotechnology Co. Ltd.) and transcribed into cDNA. Reaction mixtures were incubated for examining it with primers we need in our pathway like caspas-3, caspase-8, caspase-9, XIAP, cyclin-B and Cdk-1. The expression level of each mRNA relative to that of GAPDH was calculated using a standard curve.

\begin{tabular}{|l|l|}
\hline Primers & Sequence \\
\hline Cyclin B & 5-CAGTCAGCCAAAATACCTACCTGGGT-3, 5-ACACCAACCAGCTGCATCTTCTT-3 \\
\hline CDK1 & 5-GGATGTGCTTATGCAGGATT-3, \\
& 5-CAGCCAAAGAAAgACCAGAC-3 \\
\hline Caspase-9 & 5_-ATG GAC GAA GCG GAT CGG-3 \\
& 5_-CCC TGG CCT TAT GAT GTT-3 \\
\hline XIAP & 5-GGATTCTCCATGGCAGATTATGAAGC-3 \\
& 5'-CTCTCGAGTTACCCCATGGATCCCAGATAGTTTTCAACT-3' \\
\hline Caspase-3 & 5'-TTAATAAAGGTATCCATGGAGAACACT-3' \\
& 5'-TTAGTGATAAAAA TAGAGTTCTTTTGTGAG-3 \\
\hline Caspase-8 & 5_-CGGGATCCGCCACCATGGACTTCAGCAGAAATC-3 \\
& 5_-TCCCCGGGCACCATCAATCAGAAGGG-3 \\
\hline GAPDH & 5_-GTG GAA GGA CTC ATG ACC ACA G-3 \\
& 5_-CTG GTG CTC AGT GTA GCC CAG-3 \\
\hline
\end{tabular}

\subsection{Western Blot}

MDA-MB231 cells were treated with fucoidan and then washed once with ice-cold phosphate-buffered saline containing $1 \mathrm{mM} \mathrm{Na} \mathrm{VO}_{4}$ and extracted with lysis buffer $(50 \mathrm{mM}$ Tris, $\mathrm{pH} 8.0,150 \mathrm{mM} \mathrm{NaCl}, 5 \mathrm{mM}$ EDTA, $5 \%$ glycerol, $1 \%$ Triton X-100, $25 \mathrm{mM} \mathrm{NaF}, 2 \mathrm{mM} \mathrm{Na} \mathrm{VO}_{4}, 10 \mathrm{mg} / \mathrm{mL}$ of each aprotinin, leupeptin and pepstatin). The preparation of cytoplasmic was conducted using the NE-PER cytoplasmic extraction reagents (Pierce, Rockford, IL). Cell lysates were frozen and thawed three times and were further centrifuged at 14,000 g for $10 \mathrm{~min}$ at $4^{\circ} \mathrm{C}$ to pellet insoluble material. The supernatant of cell extracts was analyzed for protein concentration by a DC protein assay kit based on the Lowry method (Bio-Rad, Hercules, and CA). Equal amounts of protein from each sample were separated on $10 \%$ sodium dodecyl sulfate-polyacrylamide gels and transferred to PVDF membranes (MSI, Westborough, MA). Membranes were blocked in 5\% nonfat dry milk in Tris-buffered saline containing $0.05 \%$ Tween-20 (TBST) and then incubated with rabbit antibodies against caspase-3, caspase-8, caspase-9, XIAP and GAPDH, which was used as control for equal protein loading. The immunoblots were then washed three times with TBS-T buffer, incubated with a horseradish peroxidaseconjugated secondary antibody (goat anti-rabbit IgM, Santa Cruz Biotechnology), and developed using chemiluminescent substrate (Pierce). 


\subsection{Measurement of ROS}

MDA-MB231 cells $\left(2 \times 10^{6}\right.$ cells per assay $)$ were treated with fucoidan at various concentrations. Following treatment, the cells were wash once with PBS and incubated with $10 \mu \mathrm{M}$ carboxy-H 2 DCFDA, a fluorescent indicator of intracellular levels of ROS (Molecular Probe, Carlsbad, CA), for 30 minutes at $37^{\circ} \mathrm{C}$ in PBS. The cells were harvested and analyzed by flow cytometry using Flow Jo 7.6 software.

\subsection{Effect of Fucoidan on MDA-MB-231 Cells Growth}

\section{Results}

The anti-proliferative effect of fucoidan on MDA-MB-231cells was examined by exposing them to different concentrations of fucoidan for $24 \mathrm{~h}$. When MDA-MB-231 cells were exposed to fucoidan, cell growth was inhibited in a dose-dependent manner as illustrated in Fig 2. Compared to the control, 0.2 to $1.0 \mathrm{mg} / \mathrm{ml}$ of fucoidan significantly inhibited cell growth. After treatment with fucoidan for $24 \mathrm{~h}$, a dose-dependent inhibition of cell viability was observed. Therefore, fucoidan induced growth inhibition of MDA-MB-231 cell line.

\subsection{Effect of Fucoidan on MDA-MB-231Cell Cycle Distribution}

The cell cycle distribution was also examined with flow cytometry at indicated time and various doses. As shown in Fig 3, fucoidan induced a significant cell population increase in G2/M phase following $24 \mathrm{~h}$ treatment with different concentrations $(0-1.0 \mathrm{mg} / \mathrm{ml})$. As shown in table 1, the percentage of cells at G1 phase decreases, while that at $\mathrm{G} 2 / \mathrm{M}$ phase increases and apoptotic cells increases with increasing fucoidan concentration.

\subsection{Fucoidan Induces Apoptosis in MDA-MB-231}

Fucoidan treatment also caused cells to lose their phospholipid membrane asymmetry. The exposure of phosphatidylserine to the outside of the plasma membranes was detected by annexin V-FITC staining in MDAMB-231 cells. The annexin V staining results as shown in Fig 4 indicate that fucoidan causes a significant increase in the number of apoptotic cells (annexin $\mathrm{V}$ positive) in a dose-dependent manner. The effect of fucoidan $(1 \mathrm{mg} / \mathrm{mL})$ on MDA-MB-231 cells was the most significant.

\subsection{Cell Morphplogical Assessment (Hoechst Test)}

The induction of apoptosis by fucoidan in MDA-MB231 cells was confirmed in fluorescence photomicrographs of cells stained with Hoechst 33258. Fig 5 clearly demonstrates a shrunken nucleus and peripherally clumped and fragmented chromatin. These characteristics are typical of apoptotic cells.

\subsection{Effect of fucoidan on expression of the caspases and XIAP at transcriptional level in MDA-MB 231 cells}

Caspases are activated in a sequential cascade of cleavages from their inactive forms. Activation of caspase- 8 leads to the cleavage of a number of caspases, one of which is caspase-3, while XIAP could inhibit activation of caspases-3.

In order to investigate the potential involvement of caspases in the cell death induced by fucoidan, we examined the expression of XIAP which is correlated with inhibition of apoptosis and further investigated expression of those critical caspases. Our results indicated the expression of caspase- 8 , caspase- 3 and caspase- 9 was increased gradually and inversely that of XIAP ,CDK1 and cyclin-B was decreased gradually after fucoidan incubation (Fig.6).

\subsection{Effect of fucoidan on the caspases and XIAP protein expression in MDA-MB 231 cells}

To assess the role of caspases in this apoptotic process, the expression of caspase-8, caspase -3 , caspase -9 and XIAP was examined by Western blot analysis. As shown in Fig 7, the increase of caspases $(-3,-8$, and -9$)$ and decrease of XIAP both indicate the effect of fucoidan on caspase cascade.

\subsection{Effect of fucoidan on ROS generation in MDA-MB-23 cells}

Fucoidan induced oxidative damage in human breast cells and increased intracellular reactive oxygen species. We have examined activation of members of the caspase family as well as expression of mitochondrial-dependent apoptotic factors and ROS level. Our results indicate that fucoidan induces apoptosis via a caspase-8 activation pathway.

Increased levels of ROS have been demonstrated to induce depolarization of the mitochondrial membrane, which eventually produces an increase in the level of other pro-apoptotic molecules in cells[9]. With the increase of fucoidan concentration, increasing ROS level was observed in Fig.8 (from left to right side), which means that fucoidan induces apoptosis via promoting ROS accumulation. 
Fucoidan dose-dependently increased the reactive oxygen species production. MDA-MB-231 cells were treated with or without fuciodan (a-e: $0,0.2,0.4,0.6,0.8$ and $1.0 \mathrm{mg} / \mathrm{ml}$ ) for $24 \mathrm{~h}$ and then were harvested. The amount of reactive oxygen species was measured using the fluorescent probe DCFH-DA by flow cytometry.

\section{Discussion}

Fucoidan is one of the major sulfated polysaccharides of brown seaweeds whose sulfate group plays a vital role in various biological activities ${ }^{[18,}$ 19]. We used commercial available fucoidan purified from F.vesiculosus. A number of genes that are associated with apoptotic cell death were tested in order to clarify the mechanism by which fucoidan treatment induces apoptosis in MDA-MB-231 cells. According to some previously conducted studies, caspases act as key players of fucoidan-induced apoptosis ${ }^{[20-27]}$. These findings have guided the researcher to propose a model in which fucoidan promotes apoptosis in MDA-MB-231 cells through increasing caspases activity.

In this paper, MTT results domenstrated that fucoidan inhibited cell proliferation and significant cytotoxicity on MDA-MB-231cells in dose-dependent manner. Flow cytometry results further indicated that treatment of fuciodan induced G2/M phase arrest, which was due to the downregulation of the genes related to G2/M phase (cyclin B1, CDK1) in dose-dependent manner in MDA-MB231 cells. In the meanwhile this study also found the decrease of Bcl-2 level as well as the increase of Bax level in MDA-MB231 cells by fucoidan treatment (data not shown). This apoptotic effect is triggered via a pathway depending upon caspase- 3 and -8 activation.

In fact, it is known that activation of caspase- $3,-8$ and -9 cleavages by caspase- 3 are hallmarks of apoptosis in MDA-MB231 cells. Caspase-8 plays a vital role in apoptosis triggered by the interaction of ligand with death receptor as FAS and directly cleared by caspase- 8 activation which after that activate caspase -3 (Fig. 9). Caspase- 3 is active over a broad $\mathrm{pH}$ range that is slightly higher (more basic) than many of the other executioner caspases. This broad range indicates that caspase-3 will be fully active under normal and apoptotic cell conditions $^{[28]}$. XIAP binds and inhibits initiator caspase- 9 , which is directly involved in the activation of executioner caspase- ${ }^{[29]}$. During the caspase cascade, however, caspase- 3 functions to inhibit XIAP activity by cleaving caspase-9 at a specific site, preventing XIAP from being able to bind to inhibit caspase-9 activity ${ }^{[30]}$. Caspase- 3 has been found to be necessary for typical role in apoptosis, where it is responsible for chromatin condensation and DNA fragmentation ${ }^{[31]}$.

In addition, we observed that fucoidan treatment enhanced intracellular ROS levels in dose-dependent manner, suggesting that induction of oxidative stress was an important event in the cell death induced by fucoidan ${ }^{[32]}$.

\section{Conclusion}

Fucoidan inhibits the MDA-MB-231 cell proliferation by inducing cell apoptosis through activation of caspase cascade and induction of ROS, and the apoptosis is related to the activation of caspase-8, caspase-3, and caspase -9 and the inhibition of XIAP.

\section{Acknowledgements}

This work was supported by the Wuhan Municipal Science and Technology research project (Grant No. 201260523185) and the Public science and technology research funds projects of ocean (201005013).

\section{References}

[1]. Cumashi, A., et al., A comparative study of the anti-inflammatory, anticoagulant, antiangiogenic, and antiadhesive activities of nine different fucoidans from brown seaweeds. Glycobiology, 2007. 17(5): p. 541-552.

[2]. Fitton, J.H., Therapies from fucoidan; multifunctional marine polymers. Marine drugs. 9(10): p. 1731-1760.

[3]. Ye, J., et al., Enzyme-digested fucoidan extracts derived from seaweed Mozuku of Cladosiphon novae-caledoniae kylin inhibit invasion and angiogenesis of tumor cells. Cytotechnology, 2005. 47(1-3): p. 117-126.

[4]. Ermakova, S., et al., Fucoidans from brown seaweeds Sargassum hornery, Eclonia cava, Costaria costata: structural characteristics and anticancer activity. Applied biochemistry and biotechnology. 164(6): p. 841-850.

[5]. Hu, W. and J.J. Kavanagh, Anticancer therapy targeting the apoptotic pathway. The lancet oncology, 2003. 4(12): p. 721-729.

[6]. Martin, K. and J. Barrett, Reactive oxygen species as double-edged swords in cellular processes: low-dose cell signaling versus high-dose toxicity. Human \& experimental toxicology, 2002. 21(2): p. 71-75.

[7]. Scherz-Shouval, R. and Z. Elazar, Regulation of autophagy by ROS: physiology and pathology. Trends in biochemical sciences. 36(1): p. 30-38.

[8]. Acharya, A., et al., Redox regulation in cancer: a double-edged sword with therapeutic potential. Oxidative medicine and cellular longevity. 3(1): p. 23-34.

[9]. Simon, H.-U., A. Haj-Yehia, and F. Levi-Schaffer, Role of reactive oxygen species (ROS) in apoptosis induction. Apoptosis, 2000. 5(5): p. 415-418.

[10]. Schumacker, P.T., Reactive oxygen species in cancer cells: live by the sword, die by the sword. Cancer cell, 2006. 10(3): p. 175176.

[11]. Pelicano, H., D. Carney, and P. Huang, ROS stress in cancer cells and therapeutic implications. Drug Resistance Updates, 2004. 7(2): p. 97-110. 
[12]. Ashkenazi, A. and V.M. Dixit, Death receptors: signaling and modulation. Science, 1998. 281(5381): p. 1305-1308.

[13]. Wallach, D., et al., Tumor necrosis factor receptor and Fas signaling mechanisms. Annual review of immunology, 1999. 17(1): p. 331-367.

[14]. Ocal \pm a, A. and A. Pandiella, Identifying breast cancer druggable oncogenic alterations: lessons learned and future targeted options. Clinical Cancer Research, 2008. 14(4): p. 961-970.

[15]. Feuer, E.J., et al., The lifetime risk of developing breast cancer. Journal of the National Cancer Institute, 1993. 85(11): p. 892-897.

[16]. Jordan, V.C. and A.M. Brodie, Development and evolution of therapies targeted to the estrogen receptor for the treatment and prevention of breast cancer. Steroids, 2007. 72(1): p. 7-25.

[17]. Come, S.E., et al., Endocrine and targeted manipulation of breast cancer: summary statement for the Sixth Cambridge Conference. Cancer, 2008. 112(S3): p. 673-678.

[18]. Itoh, H., et al., Antitumor activity and immunological properties of marine algal polysaccharides, especially fucoidan, prepared from Sargassum thunbergii of Phaeophyceae. Anticancer research, 1993. 13(6A): p. 2045.

[19]. Riou, D., et al., Antitumor and antiproliferative effects of a fucan extracted from ascophyllum nodosum against a non-small-cell bronchopulmonary carcinoma line. Anticancer research, 1996. 16(3A): p. 1213.

[20]. Aisa, Y., et al., Fucoidan induces apoptosis of human HS-Sultan cells accompanied by activation of caspase-3 and down-regulation of ERK Pathways. American journal of hematology, 2005. 78(1): p. 7-14.

[21]. Haneji, K., et al., Fucoidan extracted from Cladosiphon okamuranus Tokida induces apoptosis of human T-cell leukemia virus type 1-infected T-cell lines and primary adult T-cell leukemia cells. Nutrition and cancer, 2005. 52(2): p. 189-201.

[22]. Hyun, J.H., et al., Apoptosis inducing activity of fucoidan in HCT-15 colon carcinoma cells. Biological and Pharmaceutical Bulletin, 2009. 32(10): p. 1760-1764.

[23]. Kim, E.J., et al., Fucoidan present in brown algae induces apoptosis of human colon cancer cells. BMC gastroenterology, 2010. 10(1): p. 96.

[24]. Koyanagi, S., et al., Oversulfation of fucoidan enhances its anti-angiogenic and antitumor activities. Biochemical pharmacology, 2003. 65(2): p. 173-179.

[25]. Nagamine, T., et al., Inhibitory effect of fucoidan on Huh7 hepatoma cells through downregulation of CXCL12. Nutrition and cancer, 2009. 61(3): p. 340-347.

[26]. Ye, J., et al., Enzyme-digested Fucoidan Extracts Derived from Seaweed Mozuku of Cladosiphon novae-caledoniaekylin Inhibit Invasion and Angiogenesis of Tumor Cells. Cytotechnology, 2005. 47(1): p. 117-126.

[27]. Yamasaki-Miyamoto, Y., et al., Fucoidan induces apoptosis through activation of caspase-8 on human breast cancer MCF-7 cells. Journal of agricultural and food chemistry, 2009. 57(18): p. 8677-8682.

[28]. Stennicke, H.R. and G.S. Salvesen, Biochemical characteristics of caspases-3,-6,-7, and-8. Journal of Biological Chemistry, 1997. 272(41): p. 25719-25723.

[29]. Li, P., D. Nijhawan, and X. Wang, Mitochondrial activation of apoptosis. Cell, 2004. 116(2 Suppl): p. S57.

[30]. Denault, J.-B., et al., Caspase 3 attenuates XIAP (X-linked inhibitor of apoptosis protein)â€"mediated inhibition of caspase 9. Biochemical Journal, 2007. 405(Pt 1): p. 11.

[31]. Porter, A.G. and R.U. Jlanicke, Emerging roles of caspase-3 in apoptosis. Cell death and differentiation, 1999. 6(2): p. 99.

[32]. Hassan, S., et al., In vitro challenge using thymoquinone on (HepG2) cell line. Toxicology Letters, 2009. 189: p. S266. 